\title{
Esophageal manifestations of bullous pemphigoid
}

\begin{abstract}
We report the case of an 86-year old female who presented with dysphagia, nausea and epigastric tenderness. She had a history of bullous pemphigoid treated with high dose steroids and immunosuppression. She was in the process of tapering her steroids following a bullous pemphigoid flare when these symptoms developed; she did not have any skin involvement at the time of presentation. She underwent endoscopy to investigate these symptoms, which showed desquamative mucosa and a trapped food bolus. An iatrogenic tear was caused in attempting to bypass the food bolus. Endoscopy was repeated in the operation room and a stricture was found at the site where a food bolus was previously seen. She was put back on high dose steroids and the stricture was dilated leading to resolution of her symptoms. Esophageal involvement of bullous pemphigoid is rare, and usually manifests as hemorrhagic bullae. An esophageal stricture resulting from bullous pemphigoid has not yet been reported.
\end{abstract}

Volume 7 Issue 4 - 202 I

\author{
Areej Adil,' Lukasz Kwapisz, ${ }^{2}$ Brian Yan ${ }^{2}$ \\ 'Department of Medicine, Memorial University of \\ Newfoundland, Canada \\ ${ }^{2}$ Department of Medicine, Western University, Canada
}

\begin{abstract}
Correspondence: Lukasz Kwapisz, Department of Medicine, Division of Gastroenterology, Western University, 800 Commissioners Rd E, London, ON N6A 5W9, Canada, Email Lukasz.kwapisz@londonhospitals.ca
\end{abstract}

Received: May 22, 2017| Published: August 22, 2017

Keywords: bullous pemphigoid, esophagus, endoscopy, stricture

\section{Introduction}

Bullous pemphigoid (BP) and mucous membrane pemphigoid (MMP) are autoimmune disorders involving $\operatorname{IgG}$ antibodies to basement membrane zone antigens. ${ }^{1}$ The natural course of these diseases is that of exacerbations and remissions. ${ }^{2}$ Older adults over the age of 60 and men are more likely to be affected. ${ }^{3}$ BP manifests as tense, $1-3 \mathrm{~cm}$ fluid filled bullae on the skin. ${ }^{2}$ The bullae may be on an erythematous base and are usually associated with pruritus. ${ }^{2} \mathrm{~A}$ prodromal phase lasting weeks to months may precede development of bullae. ${ }^{1}$ Biopsy of the edge of an intact blister is the gold standard in diagnosis of BP or MMP. Direct immunofluorescence shows linear IgG or linear C3 staining along the basement membrane zone. ${ }^{1} \mathrm{~A}$ diagnosis can also be made through serology. In BP, serology shows IgG antibodies against bullous pemphigoid antigen (BP180 or BP230), which are components of type XVII collagen. In MMP, the antibodies are variable and can include BP180 or BP230. ${ }^{1}$

BP can involve any area of the skin and $10-20 \%$ of patients have oral involvement; ocular, nasal, pharyngeal and esophageal mucosa are rarely involved. ${ }^{4}$ Mucosal involvement is more common with MMP, which manifests as mucosal inflammation and erosion. Oral mucosa is most often involved in MMP as $85 \%$ of patients with MMP have oral involvement. ${ }^{5} 24 \%$ of patients with MMP have skin involvement, and $4 \%$ have esophageal involvement. ${ }^{5}$ Scarring is one of the distinguishing features between MMP and BP as BP bullae do not leave scars, whereas MMP does. ${ }^{6}$ In this paper we report a case of esophageal BP including an esophageal stricture.

\section{Case report}

An 86 year old female with a history of Bullous Pemphigoid (BP) presented to the emergency department with a one month history of solid food dysphagia and epigastric tenderness. When she was initially diagnosed with BP, she was treated with high dose oral steroids and mycophenolate. She continued high dose steroids until two weeks after her blisters resolved, which took a few months. She then started a very slow taper.

One month prior to her presentation, she had decreased her dose of steroids as part of the tapering process. She did not have any skin lesions or oropharyngeal involvement. An upper endoscopy was completed, which showed desquamative mucosa at the $25 \mathrm{~cm}$ mark (Figure 1). There was a large food bolus distal to this, and in attempting to get past the food bolus, an iatrogenic mucosal tear was caused at the $29 \mathrm{~cm}$ mark (Figure 2). Chest x-ray and CT showed pneumomediastinum without extravasation of contrast. She was taken to the operating room three days later for a repeat upper endoscopy under general anesthesia. The repeat endoscopy showed esophagitis and desquamation at the $23 \mathrm{~cm}$ mark, a healing perforation site, and although the food bolus had cleared, a stricture was seen at the same site. This was dilated with a $11 \mathrm{~mm}$ savary dilator. Her symptoms resolved after this procedure.

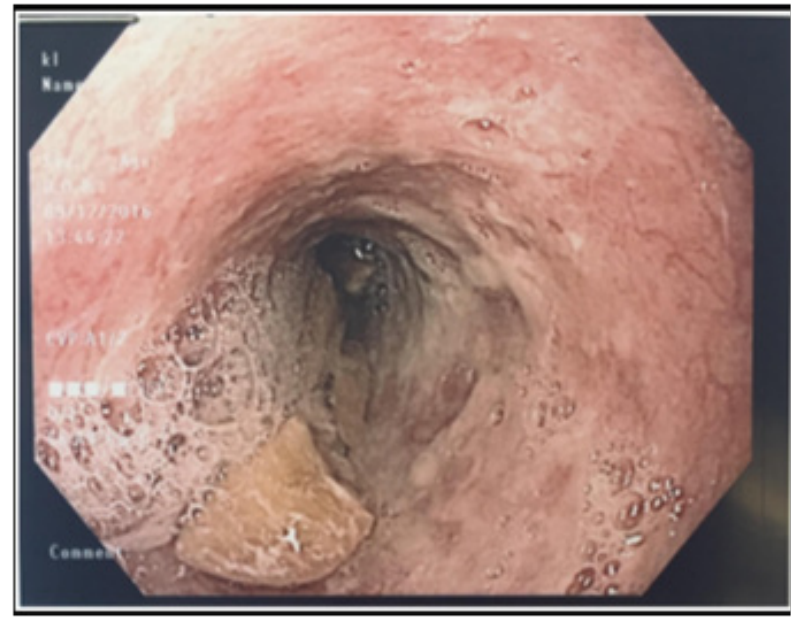

Figure I Bullous pemphigoid showing an esophageal stricture.

\section{Discussion}

Bullous pemphigoid and MMP are a part of a larger category of pemphigoid disorders. Features that distinguish BP from MMP are the area of involvement, scarring, and immunochemistry. MMP usually involves oral mucosal involvement and causes scarring. Antigens identified in MMP include BP180, BP230, Laminin 5/6, Type VII collagen, and Integrin 4 subunit, ${ }^{6}$ whereas BP involves BP180 and BP230. It is important to differentiate esophageal BP from MMP 
as the approach to treatment can differ. The approach to treatment of a patient with BP generally progresses from topical steroids, to systemic steroids to immunosuppression depending on the severity of the disease. However, a patient with extraoral MMP is treated more aggressively as the bullae cause scarring and systemic therapy is used initially regardless of the severity of the disease. ${ }^{6}$

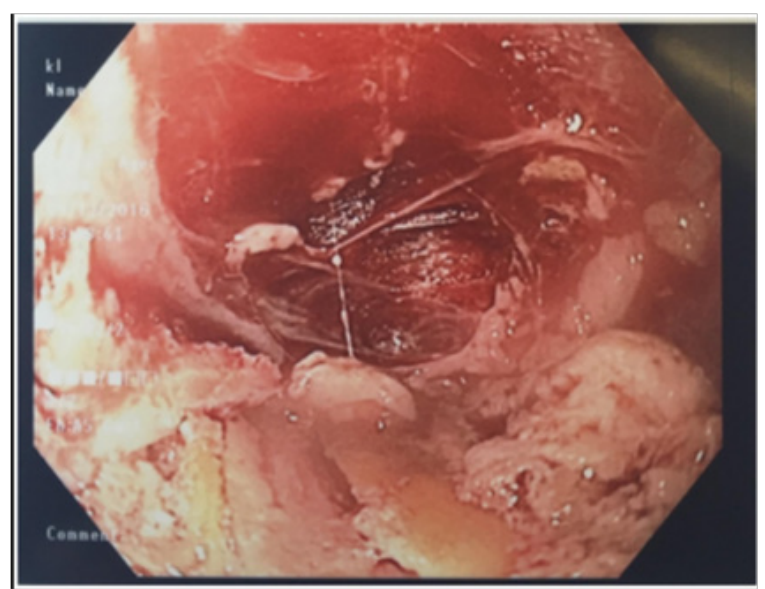

Figure 2 Appearance of iatrogenic mucosal tear.

There are few case reports of esophageal involvement in BP as it is not very common. Case reports of esophageal BP report hemorrhagic bullae in the esophagus. ${ }^{7-10}$ There has been one case report of an 18 year old female who was diagnosed with BP through biopsy and developed esophageal stenosis four years after BP was diagnosed. A repeat biopsy at the time of esophageal stenosis showed that she had converted to MMP. ${ }^{11}$ This was attributed to epitope spreading. Our patient had biopsy proven BP with skin involvement approximately one year prior to presenting with dysphagia. To our knowledge, there have not been reports of esophageal strictures in BP. Strictures may be less likely to occur in cases of BP as it does not cause scarring. Further research is required to investigate the mechanism by which BP could cause esophageal strictures and to further characterize epitope spreading in BP.

\section{Funding}

All authors disclosed no financial relationships relevant to this publication.

\section{Acknowledgements}

None.

\section{Conflicts of Interest}

There is no conflict of interest.

\section{References}

1. Schmidt E, Zillikens D. The Diagnosis and Treatment of Autoimmune Blistering Skin Diseases. Deutsches Ärzteblatt International. 2011;108(23):399-405.

2. Di Zenzo G, Marazza G, Borradori L. Bullous Pemphigoid: Physiopathology, Clinical Features and Management. Advances in Dermatology. 2007;23:257-288.

3. Jung M, Kippes W, Messer G, et al. Increased risk of bullous pemphigoid in male and very old patients: A population-based study on incidence. $J$ Am Acad Dermatol. 1999;41(2 pt 1):266-268.

4. Schmidt E, della Torre R, Borradori L. Clinical Features and Practical Diagnosis of Bullous Pemphigoid. Dermatologic Clinics. (2011);29(3): 427-438.

5. Ahmed AR, Hombal SM. Cicatricial Pemphigoid. Int J Dermatol. (1986);25(2):90-96.

6. Chan LS, Ahmed AR, Anhalt GJ, et al. The First International Consensus on Mucous Membrane Pemphigoid. Arch Dermatol. 2002;138(3):370379

7. Maharshak N, Sagi M, Santos E, et al. Oesophageal involvement in bullous pemphigoid. Clinical and Experimental Dermatology. 2012;38(3):274-275.

8. Chong VH, Lim CC, Vu C. A Rare Cause of Acute Upper Gastrointestinal Bleeding. Surg Laparosc Endosc Percutan Tech. 2006;16(2):91-93.

9. Eng TY, Hogan WJ, Jordon RE. Oesophageal involvement in bullous pemphigoid: A possible cause of gastrointestinal hemorrhage. British Journal of Dermatology. 1978;99(2):207-210.

10. Sharon P, Greene ML, Rachmilewitz D. Esophageal involvement in bullous pemphigoid. Gastrointest Endosc. 1978;24(3):122-123.

11. Sárdy M, Borovaya A, Horváth ON, et al. Successful rituximab treatment of juvenile bullous pemphigoid with esophageal scarring due to epitope spreading. J Dtsch Dermatol Ges. 2016;14(6):618-621. 